\title{
The Information and Communication Revolution: Impact on Society
}

\author{
Rudi Papa \\ "Sapienza" University of Rome, Department of Political Science \\ rudi.papa@uniroma1.it
}

\section{Doi:10.5901/mjss.2015.v6n5s1p282}

\begin{abstract}
The simple idea of connecting people through a variety of open platforms and freely editable is significantly destabilizing the common perception of concepts, such as identity, knowledge and research information. Access to the convergence between telecommunications, computer and traditional media is speeding up the cycle of news, interactivity and availability of multimedia content on the same customization of information. The new media make possible an immediate return issue sent by the receiver on the same transmission channel used by the issuer. In this new dimension, the text is not only necessarily written or sound or linked to images. Which have initiated a profound transformation. The objective of this paper is to give a point of view about the impact of information and communication technologies on the everyday life. For this paper was used mixed method approach and different number of perspectives from general to comparative one in order to enhance the research.
\end{abstract}

Keywords: Information revolution ; mass communication ;journalism; social media; connecting people;

\section{Introduzione}

La rapidità con cui circolano le informazioni nel mondo digitale, ha accentuato la domanda di notizie in tempo reale più di quanto non abbiano mai fatto le televisioni fornitrici di news 24 ore su 24. Da parte dei giornalisti ciò ha portato ad un maggiore margine di errore dovuto alla velocità con cui si è costretti a verificare una notizia, e spesso all'inserimento in agenda di eventi che in realtà sarebbero trascurabili, ma che vengono segnalati in nome della dinamicità del servizio. Anche gli editori si sono trovati in pochi anni ad affrontare una realtà in continuo mutamento, che ha richiesto, e richiede ancora, non poca capacità di adattamento. Se all'inizio sembrava logico esigere il corrispettivo di un prezzo per ogni articolo consultato dal proprio computer, col passare del tempo si è appreso che non è scontato che l'utente consideri appropriato pagare per qualcosa che può ottenere attraverso altri servizi analoghi gratuiti o attraverso altri canali comunicativi. Questa continua evoluzione la possiamo osservare anche sul piano dei linguaggi utilizzati: modi di presentare le notizie, che oggi sono molto accattivanti grazie alla presenza di immagini ben definite e file multimediali, venivano un tempo sacrificati per non appesantire le connessioni telefoniche più lente di cui disponeva la maggior parte degli utenti in passato. La maggiore confidenza degli utenti con il nuovo mezzo ha richiesto tecniche di posizionamento dei banner pubblicitari sempre più sofisticate: se prima era la home del sito il luogo principe in cui collocare gli sponsor in modo da farli notare, oggi non c'è pagina che si salvi da una qualche forma di pubblicità. Ė pratica comune, per esempio, fare in modo che i file multimediali non si aprano in un player esterno installato sul computer del utente, ma in una nuova pagina del proprio browser, dove collegamenti ipertestuali alla pagina principale, ad altri documenti, e ad altri sponsor sono sicuramente presenti. La pratica giornalistica è stata dunque rivoluzionata dalle innovazioni tecnologiche degli ultimi anni. La metamorfosi con i risvolti più interessanti, dovuta soprattutto all'essenziale natura comunicativa di internet, si ha senz'altro nel rapporto che una qualsiasi organizzazione informativa ha con il suo pubblico.

\section{Informazione e Partecipazione}

Mai come ora il pubblico, grazie alla maggiore facilità con cui può comunicare la propria opinione, ha avuto la possibilità di influenzare il giornalismo, tanto che a volte i confini tra chi fruisce dell'informazione e chi la fa diventano molto labili. Internet inoltre offre ai classici destinatari delle notizie i mezzi per poter pubblicare la propria versione dei fatti in rete, e diventare quindi dei protagonisti attivi dell'informazione. Gli stessi mezzi che sono a disposizione del pubblico li ha il giornalista. Grazie ad essi egli può interagire direttamente e in modo istantaneo con il lettore. E-mail, chat, forum e weblog sfidano il vecchio concetto di comunicazione dei massmedia one-to-many, per affiancarle quelli di una comunicazione in cui le fonti classiche dell'informazione non sono più isolate e raggiungibili solo attraverso qualche 
missiva al direttore pubblicata in ultima pagina. Vita quotidiana, sfoghi, recensioni di libri e dischi, arte, fotografia, attualità sono tutti argomenti su cui può concentrarsi la missione finale di un blog. Non è passato molto tempo da quando i blog erano un piccolo fenomeno di costume a quando invece hanno cominciato a suscitare l'interesse del mondo dell'informazione data la nuova tendenza a considerarli come una nuova forma di giornalismo indipendente. Soprattutto negli Stati Uniti, i blogger acquistano sempre una maggiore dignità: alcuni di loro, per esempio, riescono a ricevere gli accrediti per la stampa necessari per la partecipazione a determinati eventi.Le novità, non solo quelle tecnologiche, apportate dai blog informativi dal punto di vista dell'internauta, destinatario e produttore di notizie, verranno affrontate parallelamente ad un confronto tra il giornalismo tradizionale e il giornalismo personale. Le differenze e le similitudini tra informazione online e weblog informativi dedurremo gli effetti di quest'ultimi sulla prima, e il livello di integrazione dei due mondi. Le future forme d'informazione derivate dal weblog che si prospettano grazie ai nuovi sviluppi tecnologici nell' ambito della telefonia mobile e di altri dispositivi portatili in grado di prelevare dati dalla rete in qualsiasi luogo. La qualità delle informazioni scambiate tramite le decine di migliaia di newsgroup presenti in rete è molto variabile, e sfugge ad ogni controllo. Per questa ragione si deve prestare molta attenzione quando si interagisce con questo tipo di fonte a scopo informativo. Se si passa dalla parte di colui che di solito è il consumatore passivo dell'informazione, cioè sul piano del normale cittadino, possiamo vedere nei newsgroup n'altra importante funzione. I newsgroup costituiscono infatti uno di quei luoghi che alimentano il cosiddetto giornalismo pubblico o giornalismo civico, dove il cittadino non solo accede alle informazioni su un determinato argomento, ma partecipa anche alla vita della comunità, e condivide con altri soggetti interessati ciò che lo spazio sociale mediatico fa succedere. II fare informazione oggi diventa molto più difficile: oltre a saper trovare le notizie e saperle scrivere bene, chi fa informazione deve acquisire delle conoscenze specifiche nell'ambito delle sinergie, delle convergenze e interazioni ipermediali. I nuovi media rendono possibile un ritorno immediato, contemporaneo all'emissione. La fruizione di contenuti via Internet ha reso familiare l'ipertestualità multimediale. Come afferma Sergio Maistrello: "Internet è il primo grande mezzo di comunicazione globale a misura di individuo. Laddove radio, tv e giornali si indirizzano genericamente a masse di persone, dentro internet ciascuno è nodo attivo di una rete per l'esplorazione della conoscenza. E non solo: ciascuno è libero di creare".

\section{3. leri e Oggi. La Trasformazione della Comunicazione di Massa}

Il termine latino MEDIUM (plurale MEDIA) ha vari significati, tra i quali quelli di "mezzo" e di "luogo accessibile, a disposizione, visibile, pubblico". Accessibilità e visibilità sono dunque le caratteristiche dei media, cioè degli strumenti di divulgazione dell'industria culturale, quali la stampa, il cinema, la televisione .Secondo l'"Oxford Dictionary", soltanto negli anni venti si è cominciato a parlarne, ma in realtà essi hanno accompagnato la vita sociale della storia dell'umanità da sempre. Lo studio dell'arte della comunicazione orale e scritta, infatti, godeva di ampio credito nella Grecia antica e a Roma; venne poi coltivata anche durante il Medioevo e, con maggiore entusiasmo, nel rinascimento. Nel tardo Settecento, nell'ambito di questa riflessione sulla comunicazione emerse il concetto di opinione pubblica, mentre fin dall'inizio dell'Ottocento acquisirono crescente importanza le masse, che, grazie alla nascita ed alla diffusione dei quotidiani, raggiunsero la consapevolezza di far parte di un gruppo di lettori dotato di una propria coscienza nazionale. Nella prima metà del novecento, in particolare sulla scia delle due guerre mondiali, l'interesse degli studiosi si è spostato sullo studio della propaganda. II bisogno di comunicare e condividere informazioni ed emozioni, dunque, è sempre stato una costante nella storia dell'umanità, dal momento che "No man is an island". I media che sono stati utilizzati per realizzare questo scopo si sono trasformati nel corso dei secoli, divenendo sempre più efficienti, meno costosi ed in grado di raggiungere un crescente numero di persone superando le tradizionali barriere spazio-fisico-temporali. Questa trasformazione da subito fu sotto gli occhi di tutti, se Papa Pio XII, durante il discorso del 17 febbraio 1950, sente il bisogno di richiamare l'attenzione dei fedeli sul ruolo delle "forze tecniche di comunicazione", potenzialmente pericolose se non gestite con prudenza. La società moderna di oggi e sempre più influenzata dal continuo evolversi delle tecniche di comunicazione. Si sono affermati i cosiddetti new media che sono i mezzi di comunicazione più recenti e di più avanzata tecnologia (Internet, telefonia mobile, pay tv...).A ben vedere, Internet è come un grande supermarket, anzi meglio - come uno shopping mall: nella gamma dei prodotti offerti da un ipermercato deve essere presente sugli scaffali anche il prodotto di nicchia nonostante la maggioranza dei clienti andrà ad acquistare il genere industriale di largo consumo.L'allettamento nei confronti del consumatore verte proprio sulla capacità di solleticare nel potenziale acquirente quelle sensazioni di appartenenza comunitaria che fanno di ogni DOPO o DOGC una bandiera della propria identità prima ancora che un sistema per la tutela della qualità e della provenienza del prodotto.

La tecnologia digitale ha diffuso in tutti gli angoli del vivere quotidiano quell'essenziale bisogno di contatto che in passato si esplicitava nell'agorà o nella piazza, nel caffè letterario o mediante una lettera, una cartolina o una telefonata. Con i termini Web 2.0 oppure 3.0 si indica proprio questo imprecisato stadio evolutivo di Internet attraverso il quale una 
serie di tecnologie hanno abilitato e continuano a farlo la nascita di innovative modalità di comunicazione e interazione (Fig 1) , segni evidenti dell'inevitabile affermazione di nuove dinamiche culturali. Ė una disposizione che porta al centro le persone e la riscoperta del valore della relazione sociale. La semplice idea di collegare una molteplicità di persone tramite piattaforme aperte e liberamente modificabili sta sensibilmente destabilizzando la comune percezione di concetti, quali l'identità, la conoscenza e la ricerca di informazioni. Si è giunti, inoltre, alla disintegrazione del costo dell'informazione, che ha creato nuove opportunità di organizzazione della produzione, con catene di offerta che travalicano continenti e fusi orari.

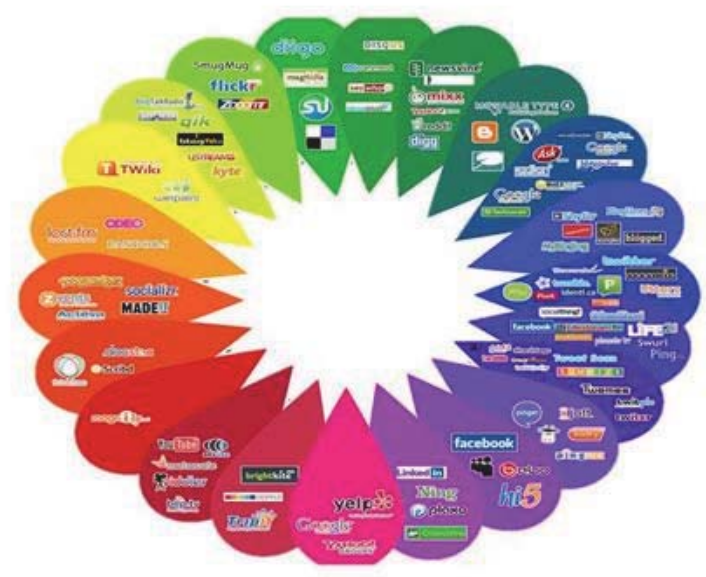

\section{Figure 1}

È quindi interessante confrontare le definizioni esistenti dei social media, incentrati sul ruolo fondamentale della comunicazione e dell'intrattenimento:

A) un sistema di strumenti, di pratiche e attività che persone e comunità di utenti utilizzano mediante applicazioni e servizi per realizzare, consumare e condividere informazioni, opinioni e conoscenze in rete. (MURERO 2010)

B) gli strumenti on-line e le piattaforme che si usano per confrontare e condividere le propie prospettive con l'altro. I social media possono assumere molte forme diverse, tra cui testi, immagini, audio e video. I social media includono blog, bacheche, podcast, wiki e vlog.( wikipedia)

C) i social media è l'uso di strumenti elettronici e di Internet con lo scopo di condividere e discutere informazioni ed esperienze con altri esseri umani

Le prime due spiegazioni risultano essere incomplete rispetto a quella elaborata dal blogger ed esperto di media Ben Parr, in quanto prendono in considerazione unicamente i sistemi online, escludendo i media elettronici. Tutte e tre, però, pongono in evidenza l'orientamento verso una partecipazione ampia da parte dei fruitori alla costruzione dell'ambiente di interazione, alla definizione dei contenuti, alla loro distribuzione e condivisione nell'ambiente sociale online.

\section{Conclusioni}

La storia della comunicazione umana vede diversi tentativi di superare il pesante vincolo delle distanze fisiche allo scopo di comunicare più velocemente. La fame di sapere tutto e subito e ormai una realtà di tutti i giorni che ci accompagna dai mezzi di trasporto ,al supermercato e perfino al bar prendendo il caffè. Negli ultimi 30 anni la diffusione dei mezzi televisivi e il sistema dei media e cambiato tantissimo imponendosi sempre di più, giorno dopo giorno. In pochi minuti una notizia, 0 un qualsiasi altro segnale multimediale possono raggiungere il più remoto punto del pianeta. Allettati dalle potenzialità del mercato online, e coscienti dell'assenza di contenuti informativi incredibili e di qualità, diversi quotidiani, a partire dalla prima metà degli anni novanta, e in coincidenza con l'invenzione del World wide web, hanno cominciato a 
proporre la versione elettronica dei loro prodotti già disponibili nelle edicole. La quantità di testate telematiche sui server di tutto il mondo e destinato a crescere esponenzialmente in modo direttamente proporzionale alla diffusione di internet nelle case. I social network,blog, twitter, facebook sono fenomeni che fino a pochi anni fa erano ristretti ad un numero piccolissimo di appassionati e esperti del campo. Invece oggi sono entrati a pieno titolo nella vita di tutti giorni. Possiamo quindi concludere dicendo che la rivoluzione e in atto e che il suo impatto e lo viviamo ogni giorno.

\section{References}

Briggs,A. Burke,P. Storia sociale dei media - Da Gutenberg a Internet, II Mulino, Bologna 2002

Carmody,S Walter Gross, Theodor H. Nelson, David Rice, and Andries van Dam. "A Hypertext Editing System for the 360" in Faiman and Nievergelt (eds.)

De Felice,L Marketing conversazionale - Dialogare con i clienti attraverso i social media e il Real-Time Web di Twitter, FriendFeed e Facebook, Gruppo24ore, Milano 2012

De Rose. Steven J. \&Van Dam Andries (1999). "Document Structure and Markup in the FRESS Hypertext System". Markup Languages 1.

Farinelli. G. [et al.], Storia del giornalismo italiano - dalle origini a oggi, UTET, Torino 2004,

Floris, G. NANNI, F. PEDACCINI, P. Fatti chiari - giornali, radio, web, talk show - come si racconta la notizia, Centro Documentazione giornalistica, Roma 2005,

Gardiner,E ,Musto.R.G. "The Electronic Book." In Suarez, Michael Felix, and H. R. Woudhuysen Oxford: Oxford University Press, 2010

Maistrello .S .Giornalismo e nuovi media, Apogeo 2010

Murero, M. Digital Literacy - Introduzione ai Social Media, libreriauniversitaria.it, Padova 2010,p 20

Pratellesi.M. New journalism. Teorie e tecniche del giornalismo multimediale Mondadori, Milano 2008

Proceedings of the Second 17 University of Illinois Conference on Computer Graphics, pp. 291-330, University of Illinois Press, 1969.

Reilly.Edwin D. Milestones in Computer Science and Information Technology. Greenwood Publishing Group, Aug , 2003.

Salarelli,A. Quando l'utente non è indipendente, il digital divide. Bit-à-brac. Informazione e biblioteche nell'era digitale, Reggio Emilia, Diabasis, 2004, 